\title{
A phase II study of concurrent nab-paclitaxel/carboplatin combined with thoracic radiotherapy in locally advanced squamous cell lung cancer
}

\author{
Kan Wu ${ }^{1}$, Lucheng Zhu ${ }^{1}$, Jiahao Wang ${ }^{1}$, Kaicheng Pan ${ }^{1}$, Bing Wang ${ }^{1}$, Xin $\mathrm{Li}^{2}$, Shaoyu Yang ${ }^{2}, \mathrm{Xiao}^{\mathrm{Xu}}{ }^{1}$, \\ Minna Zhang ${ }^{1}$, Xiadong $\mathrm{Li}^{2}$, Xueqin Chen ${ }^{2}$, Shenglin $\mathrm{Ma}^{2}$, Bing Xia, \\ ${ }^{1}$ Department of Radiation Oncology, Hangzhou Cancer Hospital, Hangzhou 310002, China; ${ }^{2}$ Department of Oncology, Affiliated Hangzhou First

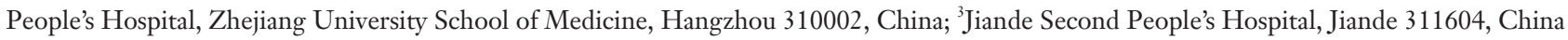 \\ Contributions: (I) Conception and design: K Wu, B Xia, S Ma; (II) Administrative support: L Zhu, J Wang; (III) Provision of study materials or \\ patients: K Wu, K Pan, B Wang; (IV) Collection and assembly of data: X Li, M Zhang, S Yang, X Li; (V) Data analysis and interpretation: K Wu, L \\ Zhu, X Chen, X Xu; (VI) Manuscript writing: All authors; (VII) Final approval of manuscript: All authors. \\ Correspondence to: Bing Xia. Department of Radiation Oncology, Hangzhou Cancer Hospital, 34 Yan-Guan Lane, Hangzhou 310002 , China. \\ Email: xb0918@hotmail.com; Shenglin Ma, MD. Department of Oncology, Affiliated Hangzhou First People's Hospital, Zhejiang University School \\ of Medicine, 261 Huansha Road, Hangzhou 310006, China. Email: mashenglin@medmail.com.cn.
}

Background: The development of chemoradiotherapy is urgently needed for locally advanced squamous cell lung cancer due to its poor prognosis and significant toxicity. Carboplatin combined with nab-paclitaxel is a useful choice as first-line therapy in advanced squamous cell lung cancer. This prospective phase II study aimed to explore the efficacy and toxicity of concurrent chemoradiotherapy with nab-paclitaxel, carboplatin, and thoracic radiotherapy in unresectable locally advanced squamous cell lung cancer.

Methods: Patients with unresectable stage III squamous cell lung cancer were eligible. All patients received nab-paclitaxel weekly at a dose of $60 \mathrm{mg} / \mathrm{m}^{2}$, in combination with carboplatin [area under the plasma concentration time curve (AUC) 2] weekly during concurrent chemoradiotherapy. Thoracic radiation was administered at a dose of $66 \mathrm{~Gy} / 33$ fractions. The consolidation chemotherapy consisted of nab-paclitaxel $\left(260 \mathrm{mg} / \mathrm{m}^{2}\right.$ on day 1 ) and carboplatin (AUC 6 on day 1 ) every 21 days was administered in two cycles after the concurrent chemoradiotherapy. The primary endpoint was objective response rate (ORR). Secondary endpoints included progression-free survival (PFS), overall survival (OS), and safety.

Results: Initially, enrollment of 21 patients was planned; however, the trial was prematurely closed due to slow recruitment. Finally, a total of 8 patients were enrolled between January 2012 and July 2015 from one institute. All patients completed concurrent chemoradiotherapy, and 6 patients (75.0\%) received consolidation chemoradiotherapy. The ORR was 75\%, with complete response (CR) 1 (12.5\%), partial remission $6(62.5 \%)$, stable disease $1(12.5 \%)$, progressive disease $1(12.5 \%)$, respectively. After a median follow-up of 15.2 (range, 2.3-51.5) months, 7 patients were dead, and 1 was alive. The median PFS and OS were 12.1 and 15.2 months, respectively. According to Common Terminology Criteria for Adverse Events version 4.0, 6 patients $(75.0 \%)$ experienced acute radiation esophagitis, $4(50.0 \%)$ were grade 2 (G2), and $2(25.0 \%)$ were $\mathrm{G} 3$; 4 patients (50\%) experienced acute radiation pneumonitis, $3(37.5 \%)$ were G2, and 1 $(12.5 \%)$ was G3. No late radiation-induced esophageal and pulmonary toxicity was observed after 1-year follow-up.

Conclusions: Concurrent nab-paclitaxel, carboplatin, and thoracic radiotherapy was shown to be an effective regimen for patients with unresectable locally advanced squamous cell lung cancer; however, further study should exercise caution due to the severe radiation esophagitis. 
Keywords: Chemoradiotherapy; nab-paclitaxel; lung squamous cell carcinoma; non-small cell lung carcinoma (NSCLC)

Submitted Mar 16, 2019. Accepted for publication Oct 10, 2019.

doi: $10.21037 /$ jtd.2019.10.81

View this article at: http://dx.doi.org/10.21037/jtd.2019.10.81

\section{Introduction}

Lung cancer is the leading cause of cancer-related death worldwide. Non-small-cell lung cancer (NSCLC) accounts for approximately $85 \%$ of all cases, and of these, about $30 \%$ are squamous cell carcinoma $(1,2)$. The locally advanced disease accounts for more than $25 \%$ of all squamous cell carcinoma (3). Concurrent platinumbased doublet chemoradiotherapy is considered the standard treatment for patients with inoperable locally advanced NSCLC with a good performance status (PS) $(4,5)$; however, the current chemotherapy regimens are not ideal due to a poor outcome $(6,7)$ and a high rate of toxicity. In recent phase III trials delivering concurrent chemoradiotherapy with a total dose between 60 and $66 \mathrm{~Gy}$, the incidence of symptomatic esophagitis ranged from $7 \%$ to $21 \%$, with corresponding rates of symptomatic radiation pneumonitis ranging from $2.5 \%$ to $7 \%(8,9)$. Therefore, the development of treatment is urgently needed to improve efficiency and reduce toxicity.

Previous studies suggest that different platinum/ third-generation chemotherapy agent combinations have similar efficacy in advanced squamous cell carcinoma (10). Recently, the choice of chemotherapy in non-squamous NSCLC has been influenced by histology. Pemetrexed has favorable efficacy and safety in non-squamous NSCLC but not in the squamous population (11). Most targeted drugs are also effective in non-squamous cell carcinoma (12-14). In locally advanced non-squamous NSCLC, the PROCLAIM trial showed less hematological toxicity for pemetrexed-cisplatin and radiation than etoposide-cisplatin and radiation. Compared with non-squamous NSCLC, treatment breakthroughs for both advanced and locally advanced squamous cell carcinoma are rare.

Albumin-bound paclitaxel is a paclitaxel formulation in which nanoparticles of paclitaxel are bound to human serum albumin. A previous study indicated that nab-paclitaxel increased intratumoral paclitaxel concentrations and antitumor activity more than cremophor based paclitaxel when an equal dose was delivered (15). Combination therapy of carboplatin and nab-paclitaxel is a useful choice as first-line therapy for patients with advanced NSCLC, especially for squamous cell cancer $(16,17)$. Weekly administration of nab-paclitaxel plus carboplatin with concurrent thoracic radiation has been reported to be a safe and well-tolerated regime for locally advanced NSCLC in a phase I study (18). Therefore, this prospective phase II study was conducted to explore the efficacy and toxicity of concurrent chemoradiotherapy with nab-paclitaxel, carboplatin, and thoracic radiotherapy in unresectable locally advanced squamous cell lung cancer.

\section{Methods}

\section{Patients}

Adults aged 18 years or older were eligible for enrolment if they had histologically/cytologically confirmed diagnosis of squamous cell lung cancer, and unresectable stage IIIA/B disease, excluding those with pericardial, pleural effusion, and those with contralateral hilar or contralateral supraclavicular lymph nodes; an Eastern Cooperative Oncology Group (ECOG) PS score of 0 or 1; a life expectancy of at least 3 months; weight loss $\leq 5 \%$ in the previous six months; adequate organ function; a measurable lesion according to Response Evaluation Criteria in Solid Tumors (RECIST), version 1.1 (19); and all detectable tumors could be encompassed by reasonable radiation therapy fields. Patients were excluded for the following reasons: complete tumor resection, recurrent disease, or disease eligible for definitive surgery; previous treatment for lung cancer; previous thoracic radiation therapy; untreated active interstitial lung disease; prior or concurrent malignancy except non-melanomatous skin cancer unless disease-free for 5 years or more; a history or presence of poorly controlled systemic diseases; history of significant neurological or mental disorder, including seizures or dementia; possibly pregnant females or males currently 
attempting to produce a pregnancy; or the volume of the whole lung receiving $\geq 20 \mathrm{~Gy}\left(\mathrm{~V}_{20}\right)$ more than $30 \%$ or the mean lung dose (MLD) of the whole lung more than 17 Gy. This study protocol was approved by our institution's ethics review board. All patients provided written informed consent before trial participation.

\section{Study design}

Patients received nab-paclitaxel weekly at a dose of $60 \mathrm{mg} / \mathrm{m}^{2}$ on day 1 , in combination with carboplatin [area under the plasma concentration time curve (AUC) 2] on day 1 for 6 cycles during concurrent chemoradiotherapy. Thoracic radiotherapy was administered on day 1 at a dose of 66 Gy/33 fractions over 6 weeks, 5 times every week. Two cycles of consolidation therapy with nab-paclitaxel $\left(260 \mathrm{mg} / \mathrm{m}^{2}\right.$ ) on day 1 and carboplatin (AUC 6$)$ on day 1 every 21 days were delivered and initiated 4 weeks after the concurrent chemoradiotherapy. Standard chemotherapy dose adjustments applied. Patients were withdrawn from the study of the adverse events failed to resolve after 14-day treatment interruption.

\section{Radiation therapy}

Patients were immobilized in the supine position with arms over the head, using a thermoplastic body frame or a vacuum pad. A free breathing contrast-enhanced helical CT scan with 5-mm slice thickness was performed in the treatment position. Use of four-dimensional computed tomography (4D-CT) during the simulation process was not mandatory. The gross tumor volume (GTV) was defined as the visualization of any gross tumor (GTV-P) and lymph nodes involved (GTV-N). If present, atelectasis was not included in the GTV and was facilitated by positron emission tomography/computed tomography (PET/CT) fusion to the planning CT. Elective node irradiation was omitted. With regard to the margin that should be added from GTV to the planning target volume (PTV) to cover the subclinical invasion and trade-off the setup error, a 1.0 - to $1.5-\mathrm{cm}$ margin was placed around the GTV-P and GTV-N, respectively. Furthermore, a kVcone beam CT (CBCT) scan was performed once a week during radiotherapy to verify the patient position. All patients were treated with Elekta Synergy linear accelerator with 6 or $10 \mathrm{MV}$ photons, and both three-dimensional conformal and intensity-modulated radiation therapy were allowed. Ninety-five percent of the PTV received 100\% of the prescribed dose, and the maximum dose of the PTV should not have exceeded $107 \%$ of the prescription dose. The maximum dose to the spinal cord was $45 \mathrm{~Gy}$, and the dose constraint for the heart was mean heart dose (MHD) $\leq 30$ Gy. For the lungs, the $V_{20}$ and $V_{5}$ were limited to $30 \%$ and $50 \%$, separately, and the MLD was $\leq 17$ Gy. The dose constraint for the esophagus was the mean dose $<34 \mathrm{~Gy}, \mathrm{~V}_{50}$ $<40 \%$, and $\mathrm{V}_{35}<50 \%$.

\section{Baseline and treatment assessments}

Baseline tumor assessments were performed within 4 weeks before the start of treatment. Subsequent tumor assessments occurred every 4 weeks during the treatment period, every 6 weeks within 24 weeks, then every 12 weeks until disease progression. Other imaging examinations, including brain magnetic resonance imaging (MRI), were performed when recurrence was suspected.

The response was assessed according to imaging examinations, which were recorded according to the RECIST criteria. The primary endpoint was objective response rate (ORR), which was defined as the percentage of participants with the best confirmed response [complete response (CR) or partial response (PR)]. Secondary endpoints included progression-free survival (PFS), overall survival (OS), and safety. OS was measured from the date of their random assignment to their death from any cause. PFS was defined as the time from the date of random assignment to objective progressive disease or death as a result of any cause. All adverse events and laboratory abnormalities were graded according to the National Cancer Institute Common Terminology Criteria for Adverse Events (NCI-CTCAE) version 4.0.

\section{Statistical analysis}

The primary endpoint of this study was the ORR. Simon's optimum two-stage phase II design was used. The study was designed to measure a response rate (CR plus PR) of $85 \%$ compared with a minimal, clinically meaningful response rate of $70 \%$, with a one-sided $\alpha$-error of 0.05 and a $\beta$-error of 0.2 . The target number of cases required in the first stage was 19 cases. Assuming a lost-to-follow-up rate of $5 \%$, at least 20 patients were needed. For the second stage, an additional 43 patients were enrolled. Therefore, a 


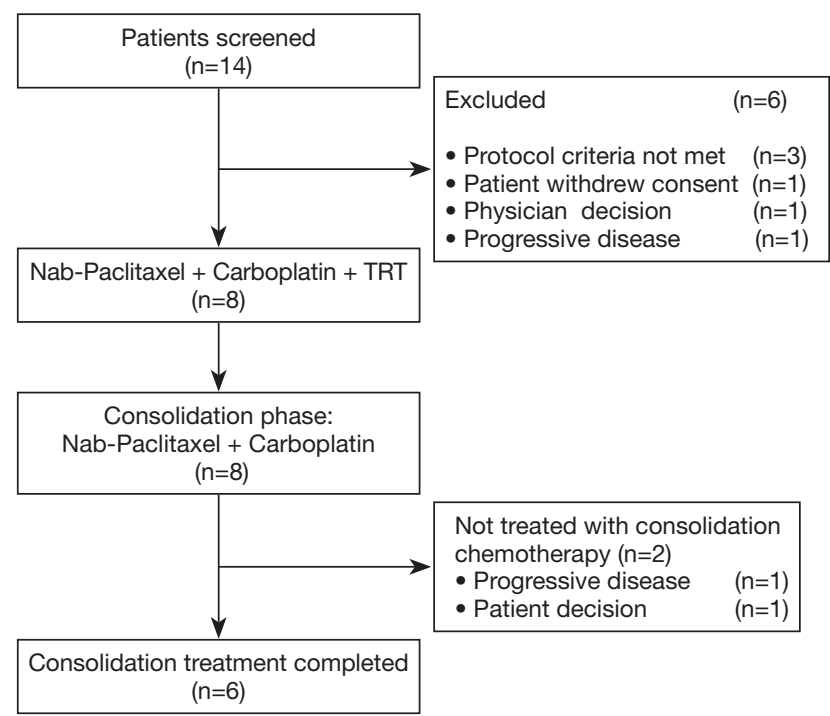

Figure 1 CONSORT diagram of the study. A total of 8 patients completed concurrent chemoradiotherapy, and 6 patients $(75.0 \%)$ received consolidation chemotherapy according to the protocol. TRT, thoracic radiotherapy.

total of 63 patients was the planned accrual size. The $t$-test was used for the statistical analysis. The survival curves were calculated using the Kaplan-Meier method. Statistical analyses were performed using SPSS version 21.0 (IBM Corp., Armonk, NY, USA). All probability values were two-sided and $\mathrm{P}$ values $<0.05$ were considered statistically significant.

\section{Results}

\section{Patient characteristics}

Initially, the enrollment of 21 patients was planned; however, the trial was prematurely closed due to slow recruitment. Finally, a total of 8 patients were enrolled between January 2012 and July 2015 from 1 institute (Figure 1). The characteristics of 8 eligible patients are listed in Table 1 . The median age was 64 years, with a range from 46 to 75 years. All of the 8 patients were male. PS was 0 in three patients and 1 in five patients. Stage distribution was as follows: IIIA, 3 patients (37.5\%); IIIB 5 patients (62.5\%).

\section{Treatment administered}

All patients completed concurrent chemoradiotherapy, and
Table 1 Patient characteristics

\begin{tabular}{lcc}
\hline Characteristics & No. & $\%$ \\
\hline No. of eligible patients & 8 & \\
Age, y & & \\
Median [range] & 64 [46-75] & \\
Gender & & \\
Male & 8 & 100.0 \\
Female & 0 & 0.0 \\
ECOG PS & & \\
0 & 3 & 37.5 \\
1 & 4 & 50.0 \\
2 & 1 & 12.5 \\
Smoking history & & \\
Absent & 2 & 25.0 \\
Present & 6 & 75.0 \\
Stage (AJCC 7th ed) & & \\
IIIA & 3 & 37.5 \\
IIIB & 5 & 62.5 \\
Location of primary site & & \\
Upper lobe & 3 & 37.5 \\
Middle lobe & 25.0 \\
Lower lobe & 3 & \\
\hline
\end{tabular}

ECOG, Eastern Cooperative Oncology Group; PS, performance status.

6 patients $(75.0 \%)$ received consolidation chemotherapy according to the protocol. Because of progressive disease (one patient) and refusal of chemotherapy (another patient) after concurrent therapy, 2 patients were unable to complete the scheduled consolidation chemotherapy.

\section{Efficacy}

By CT scan criteria, the CR and PR rate were $12.5 \%$ and $62.5 \%$, respectively, for an ORR of $75.0 \%$. Stable disease and progressive disease both occurred in 1 patient (12.5\%).

The median follow-up time of the patients was 15.2 months (range, 2.3-51.5 months). During followup, $7(87.5 \%)$ patients had died at the time of analysis. The median PFS and OS were 12.1 and 15.2 months, 
Table 2 Hematological and non-hematological adverse events according to CTCAE v4.0

\begin{tabular}{|c|c|c|c|c|c|c|}
\hline Toxicity & 0 & G1 & G2 & G3 & G4 & $\geq \mathrm{G} 3(\%)$ \\
\hline \multicolumn{7}{|l|}{ Hematological toxicities } \\
\hline Anemia & 1 & 3 & 2 & 2 & 0 & 25.0 \\
\hline Leukopenia & 2 & 1 & 1 & 4 & 0 & 50.0 \\
\hline Neutropenia & 3 & 0 & 3 & 1 & 1 & 25.0 \\
\hline Thrombocytopenia & 5 & 2 & 0 & 1 & 0 & 12.5 \\
\hline \multicolumn{7}{|l|}{ Non-hematological toxicities } \\
\hline Blood bilirubin increased & 7 & 1 & 0 & 0 & 0 & 0.0 \\
\hline ALT abnormalities & 7 & 1 & 0 & 0 & 0 & 0.0 \\
\hline AST abnormalities & 8 & 0 & 0 & 0 & 0 & 0.0 \\
\hline Creatinine increased & 8 & 0 & 0 & 0 & 0 & 0.0 \\
\hline Nausea & 5 & 1 & 1 & 1 & 0 & 12.5 \\
\hline Vomiting & 5 & 1 & 2 & 0 & 0 & 0.0 \\
\hline Anorexia & 3 & 2 & 2 & 1 & 0 & 12.5 \\
\hline Diarrhea & 6 & 2 & 0 & 0 & 0 & 0.0 \\
\hline Fatigue & 3 & 3 & 2 & 0 & 0 & 0.0 \\
\hline Neuropathy & 7 & 1 & 0 & 0 & 0 & 0.0 \\
\hline Myalgia & 7 & 1 & 0 & 0 & 0 & 0.0 \\
\hline Arthritis & 6 & 2 & 0 & 0 & 0 & 0.0 \\
\hline Radiation pneumonitis & 4 & 0 & 3 & 1 & 0 & 12.5 \\
\hline Radiation esophagitis & 2 & 0 & 4 & 2 & 0 & 25.0 \\
\hline
\end{tabular}

CTCAE, Common Terminology Criteria for Adverse Events; ALT, alanine amiotransferase; AST, aspartate transaminase.

respectively. Six $(75.0 \%)$ patients developed disease progression, 1 patient died unrelated to progression, and $1(12.5 \%)$ patient was free of disease progression as of the latest follow-up. Causes of death included the progression of the primary tumor in 4 patients $(57.1 \%)$, brain metastasis in 1 patient $(14.3 \%)$, pericardium metastasis in 1 patient (14.3\%), and bacterial pneumonia in 1 patient (14.3\%). For the patients who died of bacterial pneumonia, the time from treatment completion to onset of clinical symptoms was 14.2 months, and Klebsiella pneumoni was found in the sputum culture.

\section{Toxicity}

The acute adverse effects are summarized in Table 2. These include main toxicity (grade 3 or greater, \%): anaemia 2
(25.0\%), leukopenia 4 (50\%), neutropenia 2 (25.0\%); nausea $1(12.5 \%)$; and anorexia 1 (12.5\%). In addition, 6 patients (75.0\%) experienced symptomatic acute radiation esophagitis (ARE), $4(50.0 \%)$ were grade 2 (G2), and 2 (25.0\%) were G3; 4 patients $(50 \%)$ experienced symptomatic acute radiation pneumonitis, 3 (37.5\%) were G2, and 1 (12.5\%) was G3. Most adverse effects were grade $1 / 2$ and well-tolerated with supportive care. No late radiation-induced esophageal and pulmonary toxicity was observed after 1-year follow-up.

\section{Discussion}

The issue of how to improve local control while reducing the toxicity remains a challenge in unresectable stage III squamous cell lung cancer. Concomitant treatment with nab-paclitaxel/carboplatin and thoracic 3D conformal/ intensity-modulated radiotherapy followed by nabpaclitaxel/carboplatin consolidation chemotherapy has been shown to be an effective regimen. In the current study, the ORR was $75 \%$, and the median PFS was 12.1 months. However, the toxicity incidence, especially the incidence of ARE in the study, was higher than expected. Although toxicity did not affect the treatment completion, the incidence of severe ARE was not acceptable in this singlecenter study.

The efficacy of nab-paclitaxel/carboplatin has been reported in first-line patients with advanced NSCLC in the previous study $(16,20)$. Nab-paclitaxel is a formulation of paclitaxel which is not dissolved in Cremophor EL. This formulation potentially increases the delivery of paclitaxel into tumor cells by initiating albumin receptor (gp60)mediated transcytosis across endothelial cells (21) and accumulating drug in tumors due to the binding of albumin to secreted protein acidic and rich in cysteine (SPARC) (22).

Nab-paclitaxel has been demonstrated to be a safe drug for locally advanced NSCLC patients who received chemoradiotherapy in several phase I trials $(18,23)$. However, the incidence of ARE in the current study was high: 6 patients experienced symptomatic ARE, and twothirds of them were severe cases. The incidence of severe ARE (Radiation Therapy Oncology Group (RTOG) grade 3 or higher) was reported to be about $7-21 \%$ in NSCLC patients treated with concurrent chemoradiotherapy, and the differences in ARE risk across studies might be related to different criteria for definition of ARE, population heterogeneity, and differences in treatment $(24,25)$.

The esophagus is a critical dose constraints organ in the definitive radiotherapy of lung cancer $(26,27)$. Once 
Table 3 Dose-volume histogram parameters of esophagus and contralateral esophagus

\begin{tabular}{llcr}
\hline \multirow{2}{*}{ Characteristics } & \multicolumn{2}{c}{ Patients $(\mathrm{n}=8)$ median (range) } & $\begin{array}{c}P \\
\text { value }\end{array}$ \\
\cline { 2 - 3 } & \multicolumn{1}{c}{ Esophagus } & $\begin{array}{c}\text { Contralateral } \\
\text { esophagus }\end{array}$ & \\
\hline Dmax (Gy) & $63.05(57.60-67.94)$ & $59.44(44.40-64.92)$ & 0.73 \\
$\mathrm{~V}_{45}(\mathrm{cc})$ & $4.90(0.08-9.67)$ & $2.37(0-4.60)$ & 0.11 \\
$\mathrm{~V}_{55}(\mathrm{cc})$ & $3.75(0-7.60)$ & $1.64(0-3.47)$ & 0.12 \\
$\mathrm{~V}_{60}(\mathrm{cc})$ & $2.91(0-6.09)$ & $1.19(0-2.92)$ & 0.12 \\
$\mathrm{~V}_{60}(\%)$ & $6.62(0-12.97)$ & $5.19(0-10.67)$ & 0.61 \\
\hline
\end{tabular}

$\mathrm{Vn}$, volume of lung receiving at least $\mathrm{n}$ Gy of radiation dose.

symptomatic ARE occurs, it typically influences the quality of life and following treatment, possibly leading to poor outcomes. RTOG 0617 showed that maximum esophagitis grade was found to be an independent prognostic factor predicting OS (8). Previous studies have found a correlation between esophagitis and dosimetric parameters. A metaanalysis involving 1,082 locally advanced NSCLC patients who underwent concurrent chemoradiation therapy found that $V_{60}$ of the esophagus was the best predictor of grade $\geq 2$ and grade $\geq 3$ esophagitis (27). Another analysis reported a maximum dose exceeding 55 Gy as predictive. In this study, the incidence of severe ARE was up to $25 \%$. The dosimetric parameters of esophagitis in this study were not satisfactory, as the median maximum dose and $\mathrm{V}_{60}$ of the esophagus was 63.05 Gy and $6.62 \%$, respectively (Table 3). In fact, the specific histology of enrolled patients may be a critical reason for the high occurrence of esophagitis. Squamous cell lung tumors are usually located close to the esophagus, which may be responsible for the high radiation dose of the esophagus and the high incidence of esophagitis.

Therefore, it is important to devise new strategies for improving esophageal sparing and ultimately minimize the rates of severe ARE in squamous cell lung cancer patients undergoing concurrent chemoradiotherapy. In a recent secondary analysis, the RTOG 0617 group reported that patients treated with intensity-modulated radiotherapy had significantly lower rates of severe pneumonitis and cardiac doses compared with those treated with $3 \mathrm{D}$-conformal treatment (28). It is possible that intensity-modulated radiotherapy may be a fine treatment option for reducing the risk of esophagitis. A recent study (29) applied intensity- modulated radiotherapy-based contralateral esophageal (CE) (which was defined as the cross-sectional half of the esophagus that was opposite to the gross tumor) sparing technique (CEST), to spare the CE wall in patients with locally advanced lung cancer treated with concurrent chemoradiotherapy; the result demonstrated an improvement in esophageal toxicity with the use of this technique. The CE had more favorable dosimetry in comparison with the whole esophagus with CEST: the median maximum dose to the $\mathrm{CE}$ was significantly lower than the maximum dose to the esophagus (62.3 vs. 67.5 Gy); the median esophageal $\mathrm{V}_{60}$ was higher than the median CE $\mathrm{V}_{60}(5.3 \%$ vs. $0.5 \%)$. In contrast, there was no statistically significant difference in the median maximum dose $(63.05$ vs. 59.44 Gy, respectively; $\mathrm{P}>0.05)$ and median $\mathrm{V}_{60}(6.62 \%$ vs. $5.19 \%$, respectively; $\mathrm{P}>0.05$ ) between the esophagus and CE in the current study, which suggests no steep dose falloff across sections of the esophagus with standard esophageal dose constraints radiotherapy. Furthermore, the suggested CE dose constraints was V5 $<0.5 \mathrm{cc}$ when CEST was used (29), which was significantly lower than the dose of CE in this study (Table 3). Therefore, we tried to apply this technique to spare the $\mathrm{CE}$ wall in a patient who experienced grade $2 \mathrm{ARE}$, with the goal of decreasing the dose to the esophagus. The maximum dose to the CE was significantly decreased with CEST than with common technique (52.21 vs. $64.23 \mathrm{~Gy}$, respectively). Similarly, the maximum dose and $\mathrm{V}_{60}$ to the $\mathrm{CE}$ was significantly lower than that to the esophagus (52.21 vs. $67.74 \mathrm{~Gy} ; 0$ vs. 4.9\%, respectively) (Figure 2). It is believed that the improvement in dosimetry of CE may result in a decrease in the incidence of esophagitis. In the future, to decrease the incidence and severity of ARE, CEST may be a promising technique to treat high-risk patients with a central-location tumor.

\section{Conclusions}

This study suggests that concurrent nab-paclitaxel, carboplatin, and thoracic radiotherapy is an effective regime in unresectable locally advanced squamous cell lung cancer. However, the incidence of ARE was not acceptable in this study. A prospective trial of concurrent thoracic radiotherapy with CEST and nab-paclitaxel/carboplatin is further needed to validate the safety of this regimen. Moreover, this study is relatively small, and more data from randomized trials are needed in the future. 


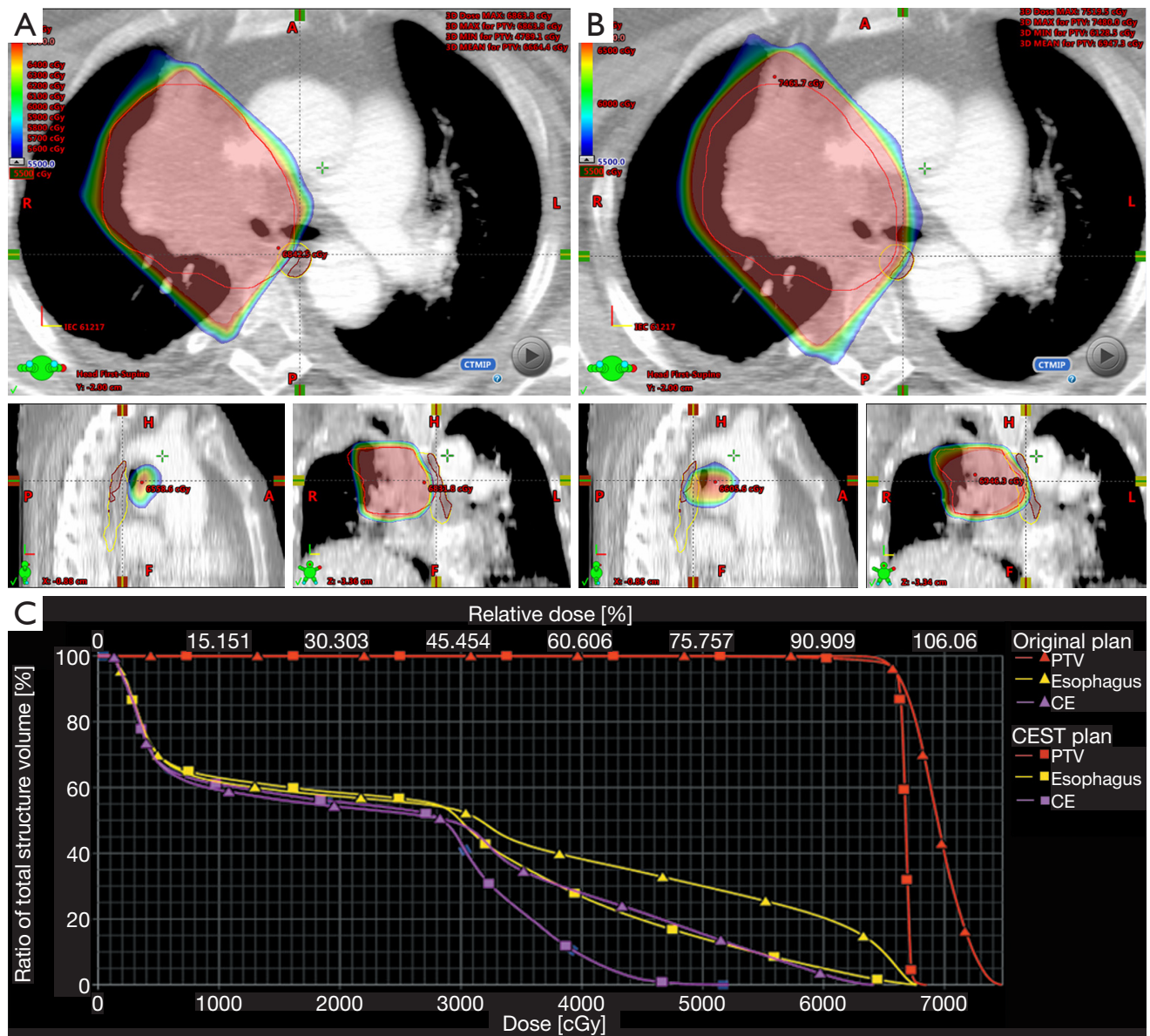

Figure 2 A shows the original plan: the PTV is covered by 66 Gy, and the contralateral esophagus received a high dose; B shows the dose distribution of the same patient using the contralateral esophageal sparing technique: the PTV is well covered by the prescription dose of $66 \mathrm{~Gy}$, and the high isodose lines are shown to effectively bend around the contralateral esophagus. C shows a dose-volume histogram of the two plans. PTV, planning target volume.

\section{Acknowledgments}

Funding: This work was supported by the Zhejiang Provincial Medicine and Health Science Foundation, China (2017KY534 and 2012KYB154) and Medical and Scientific Research Projects of Hangzhou, China (20130733Q03) for the design of the study and interpretation of data.

\section{Footnote}

Conflicts of Interest: The results of this study were presented at the meeting of 17th World Conference of Lung Cancer, December 4-7, 2016, Vienna, Austria.

Ethical Statement: The authors are accountable for all aspects of the work in ensuring that questions related to the accuracy or integrity of any part of the work are appropriately investigated and resolved. The study was approved by the institutional ethics committee of medical ethics committee of Hangzhou First People's Hospital (No. 2011-1025).

\section{References}

1. Jemal A, Bray F, Center MM, et al. Global cancer statistics. CA Cancer J Clin 2011;61:69-90.

2. Siegel R, Naishadham D, Jemal A. Cancer statistics, 2013. CA Cancer J Clin 2013;63:11-30.

3. Morgensztern $\mathrm{D}, \mathrm{Ng} \mathrm{SH}, \mathrm{Gao} F$, et al. Trends in stage distribution for patients with non-small cell lung cancer: A National Cancer Database survey. J Thorac Oncol 
2010;5:29-33.

4. Bezjak A, Temin S, Franklin G, et al. Definitive and adjuvant radiotherapy in locally advanced nonsmallcell lung cancer: American Society of Clinical Oncology clinical practice guideline endorsement of the American Society for Radiation Oncology evidence-based clinical practice guideline. J Clin Oncol 2015;33:2100-5.

5. Vansteenkiste J, De Ruysscher D, Eberhardt WE, et al. Early and locally advanced non-small-cell lung cancer (NSCLC): ESMO Clinical Practice Guidelines for diagnosis, treatment and follow-up. Ann Oncol 2013;24:vi89-98.

6. Yamamoto N, Nakagawa K, Nishimura Y, et al. Phase III study comparing second- and third-generation regimens with concurrent thoracic radiotherapy in patients with unresectable stage III non-small-cell lung cancer: West Japan Thoracic Oncology Group WJTOG0105. J Clin Oncol 2010;28:3739-45.

7. Curran WJ Jr, Paulus R, Langer CJ, et al. Sequential vs. concurrent chemoradiation for stage III non-small cell lung cancer: randomized phase III trial RTOG 9410. J Natl Cancer Inst 2011;103:1452-60.

8. Bradley JD, Paulus R, Komaki R, et al. Standard-dose versus high-dose conformal radiotherapy with concurrent and consolidation carboplatin plus paclitaxel with or without cetuximab for patients with stage IIIA or IIIB non-small-cell lung cancer (RTOG 0617): a randomised, two-by two factorial phase 3 study. Lancet Oncol 2015;16:187-99.

9. Ahn JS, Ahn YC, Kim JH, et al. Multinational randomized phase III trial with or without consolidation chemotherapy using docetaxel and cisplatin after con-current chemoradiation in inoperable stage III non-small-cell lung cancer: KCSG-LU05-04. J Clin Oncol 2015;33:2660-6.

10. Schiller JH, Harrington D, Belani CP, et al. Eastern Cooperative Oncology Group Comparison of four chemotherapy regimens for advanced non-small-cell lung cancer. N Engl J Med 2002;346:92-8.

11. Scagliotti GV, Parikh P, von Pawel J, et al. Phase III study comparing cisplatin plus gemcitabine with cisplatin plus pemetrexed in chemotherapy-naive patients with advanced-stage non-small-cell lung cancer. J Clin Oncol 2008;26:3543-51.

12. Sandler A, Gray R, Perry MC, et al. Paclitaxel-carboplatin alone or with bevacizumab for non-small-cell lung cancer. N Engl J Med 2006;355:2542-50.

13. Mok TS, Wu YL, Thongprasert S, et al. Gefitinib or carboplatin-paclitaxel in pulmonary adenocarcinoma. $\mathrm{N}$
Engl J Med 2009;361:947-57.

14. Kwak EL, Bang YJ, Camdge DR, et al. Anaplastic lymphoma kinase inhibition in non-small-cell lung cancer. N Engl J Med 2010;363:1693-703.

15. Desai N, Trieu V, Yao Z, et al. Increased antitumor activity, intratumor paclitaxel concentrations, and endothelial cell transport of cremophor-free, albumin-bound paclitaxel, ABI-007, compared with cremophor-based paclitaxel. Clin Cancer Res 2006;12:1317-24.

16. Socinski MA, Bondarenko I, Karaseva NA, Makhson AM, et al. Weekly nab-paclitaxel in combination with carboplatin versus solvent-based paclitaxel plus carboplatin as first-line therapy in patients with advanced non-smallcell lung cancer: final results of a phase III trial. J Clin Oncol 2012;30:2055-62.

17. Yang JJ, Huang C, Chen GY, et al. A randomized phase II clinical trial of nab-paclitaxel and carboplatin compared with gemcitabine and carboplatin as first-line therapy in locally advanced or metastatic squamous cell carcinoma of lung. BMC Cancer 2014;14:684.

18. Keedy VL, Lu B, Horn L, et al. A phase I study of nabpaclitaxel $(\mathrm{nP})$ with carboplatin $(\mathrm{C})$ and thoracic radiation (TRT) in patients with locally advanced NSCLC. J Clin Oncol 2011;29:7046.

19. Eisenhauer EA, Therasse P, Bogaerts J, et al. New response evaluation criteria in solid tumours: revised RECIST guideline (version 1.1). Eur J Cancer 2009;45:228-47.

20. Reynolds C, Barrera D, Jotte R, et al. Phase II trial of nanoparticle albumin-bound paclitaxel, carboplatin, and bevacizumab in first-line patients with advanced nonsquamous non-small cell lung cancer. J Thorac Oncol 2009;4:1537-43.

21. John TA, Vogel SM, Tiruppathi C, et al. Quantitative analysis of albumin uptake and transport in the rat microvessel endothelial monolayer. Am J Physiol Lung Cell Mol Physiol 2003;284:L187-96.

22. Schiemann BJ, Neil JR, Schiemann WP. SPARC inhibits epithelial cell proliferation in part through stimulation of the transforming growth factor-signaling system. Mol Biol Cell 2003;14:3977-88.

23. Lammers PE, Lu B, Horn L, et al. Nab-Paclitaxel in Combination with Weekly Carboplatin with Concurrent Radiotherapy in Stage III Non-Small Cell Lung Cancer. Oncologist 2015;20:491-2.

24. Uyterlinde W, Chen C, Kwint M, et al. Prognostic parameters for acute esophagus toxicity in intensity modulated radiotherapy and concurrent chemotherapy for locally advanced non-small cell lung cancer. Radiother 
Oncol 2013;107:392-7.

25. Wijsman R, Dankers F, Troost EG, et al. Multivariable normal-tissue complication modeling of acute esophageal toxicity in advanced stage non-small cell lung cancer patients treated with intensity-modulated (chemo-) radiotherapy. Radiother Oncol 2015;117:49-54.

26. Werner-Wasik M, Paulus R, Curran WJ Jr, et al. Acute esophagitis and late lung toxicity in concurrent chemoradiotherapy trials in patients with locally advanced non-small-cell lung cancer: analysis of the radiation therapy oncology group (RTOG) database. Clin Lung Cancer 2011;12:245-51.

27. Palma DA, Senan S, Oberije C, et al. Predicting

Cite this article as: $\mathrm{Wu} \mathrm{K}$, Zhu L, Wang J, Pan K, Wang B, Li X, Yang S, Xu X, Zhang M, Li X, Chen X, Ma S, Xia B. A phase II study of concurrent nab-paclitaxel/carboplatin combined with thoracic radiotherapy in locally advanced squamous cell lung cancer. J Thorac Dis 2019;11(11):4529-4537. doi: 10.21037/ jtd.2019.10.81 esophagitis after chemoradiation therapy for non-small cell lung cancer: an individual patient data meta-analysis. Int J Radiat Oncol Biol Phys 2013;87:690-6.

28. Chun SG, Hu C, Choy H, et al. Impact of IntensityModulated Radiation Therapy Technique for Locally Advanced Non-Small-Cell Lung Cancer: A Secondary Analysis of the NRG Oncology RTOG 0617 Randomized Clinical Trial. J Clin Oncol 2017;35:56-62.

29. Al-Halabi H, Paetzold P, Sharp GC, et al. A contralateral esophagus-sparing technique to limit severe esophagitis associated with concurrent high-dose radiation and chemotherapy in patients with thoracic malignancies. Int J Radiat Oncol Biol Phys 2015;92:803-10. 